\title{
ЧИСЛЕННОЕ ИССЛЕДОВАНИЕ ВЛИЯНИЯ ОБЪЕМНОГО СОДЕРЖАНИЯ ДИСПЕРСНОЙ КОМПОНЕНТЫ ГАЗОВЗВЕСИ НА ИНТЕНСИВНОСТЬ МЕЖФАЗНОГО СКОРОСТНОГО СКОЛЬЖЕНИЯ ПРИ РАЗЛЕТЕ ГАЗОВЗВЕСИ В ВАКУУМ
}

\author{
Д. А. Тукмаков \\ Статъя представлена членом редакционной коллегии Ю. П. Вирченко \\ ИММ - обособленное структурное подразделение ФИЦ КазНЦ РАН, \\ г. Казань, 420111, Россия \\ E-mail: tukmakovDA@imm.knc.ru
}

\begin{abstract}
Аннотация. В данной работе численно моделируется процесс истечения газовзвеси в вакуум. Математическая модель учитывала вязкость, сжимаемость и теплопроводность несущей среды, межкомпонентное силовое взаимодействие, включавшее в себя силу Стокса, динамическую силу Архимеда, силу присоединенных масс, также математическая модель учитывала межкомпонентный теплообмен. Численное решение осуществлялось при помощи явного конечно-разностного метода, с последующим применением схемы нелинейной коррекции численного решения. Целью работы было выявление влияния объемного содержания дисперсной фазы при одинаковой дисперсности и плотности материала твердой компоненты смеси, газовзвеси на интенсивность скоростного скольжения при разлете неоднородной среды в вакуум. Также были выявлены отличия в процессах истечения однородного вязкого газа и гетерогенной смеси.
\end{abstract}

Ключевые слова: математическое моделирование физических процессов, многофазные среды, уравнение Навье-Стокса, межфазное взаимодействие.

Благодарности: Работа выполнена при финансовой поддержке РФФИ Грант №19-01-00442.

Для цитирования: Тукмаков Д. А. 2020. Численное исследование влияния объемного содержания дисперсной компоненты газовзвеси на интенсивность межфазного скоростного скольжения при разлете газовзвеси в вакуум. Прикладная математика \& Физика. 52(1): 41-50. DOI 10.18413/2687-0959-2020-52-1-41-50.

\section{NUMERICAL STUDY OF THE INFLUENCE OF VOLUME CONTENT OF A DISPERSED GAS-SUSPENSION COMPONENT ON THE INTENSITY OF AN INTERPHASIC SPEED SLIDING DURING GAS-SUSPENSION SPLIT IN VACUUM}

\author{
D. A. Tukmakov \\ Article submitted by a member of the editorial board Yu. P. Virchenko \\ IME - Subdivision of FIC KazanSC of RA, \\ Kazan, 420111, Russia \\ E-mail: tukmakovDA@imm.knc.ru \\ Received March 16, 2020
}

\begin{abstract}
In this work, the process of gas suspension flowing into vacuum numerically modeled. The mathematical model took into account the viscosity, compressibility and thermal conductivity of the carrier medium, the intercomponent force interaction, which included the Stokes force, the dynamic Archimedes force, the strength of the attached masses, and the mathematical model took into account the intercomponent heat transfer. The numerical solution carried out using an explicit finite-difference method, followed by the application of a nonlinear correction scheme for the numerical solution. The aim of the work was to identify the effect of the volumetric content of the dispersed phase, with the same dispersion and density of the material of the solid component of the mixture, gas suspension on the rate of slip during expansion of an inhomogeneous medium into vacuum. Differences in the processes of the expiration of a homogeneous viscous gas and a heterogeneous mixture it also revealed.
\end{abstract}

Key words: mathematical modeling of physical processes, multiphase media, Navier-Stokes equation, interphase interaction.

Acknowledgements: The work is supported by the Russian Founda tion for Basic Research Grant N 19-01-00442.

For citation: Tukmakov D. A. 2020. OUmerical study of the influence of volume content of a dispersed gassuspension component on the intensity of an interphasic speed sliding during gas-suspension split in vacuum . Applied Mathematics \& Physics. 52(1): 41-50 (in Russian). DOI 10.18413/2687-0959-2020-52-1-41-50. 
Введение. Одним из развивающихся разделов механики жидкости и газа является динамика неоднородных сред. В монографиях [Нигматулин 1988, Кутушев, 2003, Федоров и др., 2015] разрабатывались методы математического моделирования процессов механики и теплофизики неоднородных сред. Исследованию физических эффектов в различных течениях неоднородных сред посвящен ряд публикаций в периодических изданиях [Губайдуллин, Тукмаков, 2014; Садин 2016; Федоров и др., 2016; Арсланова и др., 2016; Нигматулин и др., 2016; Тукмаков, Тукмаков, 2018; Кашапов и др., 2018; Kashapov at all, 2018; Тукмаков, 2019; Пахомов, Терехов, 2018; Azanov, Osiptsov, 2017; Вараксин, 2014]. Объектом исследований являются течения неоднородных сред с одинаковым или различным агрегатным состоянием компонент - гомогенные и гетерогенные смеси [Teklaya and all, 2015; Saikat, Meheboob, 2017; Zhuoqing, Jesse, 2019; Imran and all, 2018; Mehrabadi and all, 2015; Zhengbiao and all, 2017; Garcsia-Trianes and all, 2018; Lopez and all, 2016]. Моделирование течений неоднородных сред может быть осуществлено с помощью «равновесного» подхода - описание течения неоднородной среды как однородной жидкости или газа с введением коэффициентов, дающих поправку на физическую неоднородность среды [Нигматулин 1987]. Более сложным является «континуальный» подход, в котором для каждой из компонент смеси решается полная система уравнений динамики сплошной среды [Нигматулин 1987; Кутушев 2003; Федоров и др. 2015]. В течениях неоднородных сред, в которых объемные содержания компонент смеси имеют близкое значение, наиболее важной задачей является учёт межкомпонентного взаимодействия, определяющего динамику всей смеси в целом [Нигматулин 1987; Кутушев 2003]. В данной работе динамика газовой взвеси твердых частиц - запыленной среды описывается на основе двухскоростной, двухтемпературной модели, учитывающей межкомпонентный теплообмен, а также межкомпонентное силовое взаимодействие, включающее в себя силу Стокса, динамическую силу Архимеда и силу присоединённых масс [Нигматулин 1987; Кутушев 2003]. Одним из важных параметров течения гетерогенных смесей является скоростное скольжение фаз смеси - отличие скоростей несущей среды и дисперсной примеси. В данной работе с помощью вычислительных экспериментов исследуется то, как величина объемного содержания дисперсной фазы, при условии, что размер частиц и плотность материала твердой компоненты смеси одинаковы, влияет на интенсивность скоростного скольжения.

2. Методы исследования. Движение несущей среды описывается одномерной системой уравнений Навье-Стокса для сжимаемого теплопроводного газа с учетом межфазного силового взаимодействия и теплообмена [Кутушев 2003, Нигматулин и др., 2016]:

$$
\begin{gathered}
\frac{\partial \rho_{1}}{\partial t}+\frac{\partial\left(\rho_{1} u_{1}\right)}{\partial x}=0 \\
\frac{\partial\left(\rho_{1} u_{1}\right)}{\partial t}+\frac{\partial}{\partial x}\left(\rho_{1} u_{1}^{2}+p-\tau\right)=F+\alpha \frac{\partial p}{\partial x}, \\
\frac{\partial e_{1}}{\partial t}+\frac{\partial}{\partial x}\left(\left[e_{1}+p-\tau\right] u_{1}-\lambda \frac{\partial T_{1}}{\partial x}\right)=-Q-|F|\left(u_{1}-u_{2}\right)+\alpha\left(\frac{\partial\left(p u_{1}\right)}{\partial x}\right), \\
p=(\gamma-1)\left(e_{1}-\rho_{1}\left(u_{1}^{2}\right) / 2\right), \\
\tau=\frac{4}{3} \mu \frac{\partial u_{1}}{\partial x} .
\end{gathered}
$$

Динамика дисперсной фазы описывается уравнением сохранения «средней плотности» - произведения физической плотности материала частиц и объемного содержания дисперсной фазы, изменяющегося на различных участках физической области вместе с движением твердых частиц; уравнениями сохранения импульса и уравнением сохранения энергии, записанными с учетом теплообмена, обмена импульсом с несущей фазой:

$$
\begin{gathered}
\frac{\partial \rho_{2}}{\partial t}+\frac{\partial \rho_{2} u_{2}}{\partial x}=0 \\
\frac{\partial\left(\rho_{2} u_{2}\right)}{\partial t}+\frac{\partial}{\partial x}\left(\rho_{2} u_{2}^{2}\right)=-F-\alpha \frac{\partial p}{\partial x}, \\
\frac{\partial e_{2}}{\partial t}+\frac{\partial}{\partial x}\left(e_{2} u_{2}\right)=N u_{12} \frac{6 \alpha}{(2 r)^{2}} \lambda\left(T_{1}-T_{2}\right), \\
\rho_{2}=\alpha \rho_{20}, e_{1}=\rho_{2} C_{p} T_{2} .
\end{gathered}
$$

Здесь p, $\rho_{1}, u_{1}$ - давление, плотность, скорость несущей среды; $T_{1}, e_{1}$-- температура и полная энергия газа; $\rho_{2}, T_{2}, e_{2}, u_{2}$-- средняя плотность, температура, внутренняя энергия, скорость дисперсной фазы. Температура несущей среды находится из уравнения $(\gamma-1)\left(e / \rho-0.5 u^{2}\right) / R$, где $\mathrm{R}-$ газовая постоянная несущей фазы. Силовое взаимодействие несущей и дисперсной фазы учитывает 
силу Стокса, динамическую силу Архимеда и силу присоединенных масс. Математическая модель предполагает монодисперсный состав твердой фазы газовзвеси - все частицы имеют одинаковый размер и одинаковые физические свойства- плотность и теплоемкость материала. Внутренняя энергия взвешенной в газе дисперсной фазы определяется как $e_{1}=\rho_{1} C_{p} T_{2}$, где - удельная теплоемкость единицы массы вещества из которого состоят частицы. В уравнение энергии для несущей фазы входит тепловой поток между несущей и дисперсной фазой $Q=6 \alpha N u_{12} \lambda\left(T_{1}-T_{2}\right) /(2 r)^{2}$. В данной работе при описании межфазного силового взаимодействия -- F учитывалась сила аэродинамического сопротивления, динамическая сила Архимеда, а также сила присоединенных масс [Нигматулин, 1987; Кутушев, 2003]:

$$
\begin{gathered}
F=\frac{3 \alpha}{8 r} C_{d 2} \rho_{1}\left|u_{1}-u_{2}\right|\left(u_{1}-u_{2}\right)+\alpha \rho_{1}\left(\frac{\partial u_{1}}{\partial t}+u_{1} \frac{\partial u_{1}}{\partial x}\right)+ \\
+0.5 \alpha \rho_{1}\left(\frac{\partial u_{1}}{\partial t}+u_{1} \frac{\partial u_{1}}{\partial x}-\frac{\partial u_{2}}{\partial t}-u_{2} \frac{\partial u_{2}}{\partial x}\right)
\end{gathered}
$$

Параметры межфазного взаимодействия описаны в работе [Кутушев, 2003]:

$$
\begin{gathered}
C_{d 2}=C_{d 2}^{0} \phi\left(M_{12}\right) \varphi(\alpha), C_{d 2}^{0}=\frac{24}{R e_{12}}+\frac{4}{R e_{12}^{0.5}}+0.4, \\
\phi\left(M_{12}\right)=1+\exp \left(-\frac{-0.427}{M_{12}^{0.63}}\right), \varphi(\alpha)=(1-\alpha)^{-2.5}, \\
R e=\rho_{1} u_{1} D / \mu, R e_{12}=r \rho_{1}\left|u_{1}-u_{2}\right| / \mu, M_{12}=\left|u_{1}-u_{2}\right| / c, \operatorname{Pr}_{1}=c_{p 1} \mu(\lambda)^{-1}, \\
N u_{12}=2 \exp \left(-M_{12}\right)+0 / 459 \operatorname{Re}_{12}^{0.55} \operatorname{Pr}_{1}^{0.33}, 0<M_{12}<2,0<R e<2 * 10^{5} .
\end{gathered}
$$

Здесь $D$-- характерный размер системы.

Система уравнений математической модели решалась явным методом Мак-Кормака второго порядка [Fletcher 1988] с последующим применением схемы нелинейной коррекции решения [Музафаров, Утюжников, 1993].

Система уравнений (1)-(6) может быть записана в матричном виде:

$$
\begin{gathered}
\frac{\partial q}{\partial t}+\frac{\partial E}{\partial x}=H \\
\mathbf{q}=\left[\begin{array}{c}
\rho_{1} \\
\rho_{2} \\
\rho_{1} u_{1} \\
\rho_{2} u_{2} \\
e_{1} \\
e_{2}
\end{array}\right] \\
\mathbf{E}=\left[\begin{array}{c}
\rho_{1} u_{1} \\
\rho_{2} u_{2} \\
\rho_{1} u_{1}^{2}+p-\tau \\
\rho_{2} u_{2}^{2} \\
+\tau) u_{1}-\lambda \frac{\partial T_{1}}{\partial x} \\
e_{2} u_{2} \\
0 \\
0 \\
\left(e_{1}+p-\alpha \frac{\partial p}{\partial x}\right. \\
-F+\alpha \frac{\partial p}{\partial x} \\
F-u \frac{\partial p u_{1}}{\partial x} \\
Q
\end{array}\right]
\end{gathered}
$$

Алгоритм явного конечно-разностного метода Мак-Кормака для нелинейной системы (7) может быть записан в виде:

$$
\begin{gathered}
q_{j}^{*}=q_{j}^{n}-\frac{\Delta t}{\Delta x}\left(E_{j+1}^{n}-E_{j}^{n}\right)+\Delta t H_{j}^{n}, \\
q_{j}^{n+1}=0.5\left(q_{j}^{*}+q_{j}^{n}\right)-0.5 \frac{\Delta t}{\Delta x}\left(E_{j}^{n}-E_{j-1}^{n}\right)+0.5 \Delta t H_{j}^{n} .
\end{gathered}
$$


Монотонность решения достигалась с помощью применения схемы нелинейной коррекции вдоль пространственных направлений х и у по индексам j,k соответсвенно к компонентам вектора независимых переменных $\mathbf{q}, \mathbf{q}=\left(\rho_{1}, \rho_{2}, \rho_{1} u_{1}, \rho_{2} u_{2}, e_{1}, e_{2}\right)$. Пусть $Z_{j, k}^{n}$ - произвольная независимая функция на $n$-ом веременном слое в узле $j, k$. Тогда алгоритм корекции имел бы следующий вид:

$$
Z_{j, k}^{n *}=Z_{j, k}^{n}+\kappa\left(\delta Z_{j+1 / 2, k}^{n}-\delta Z_{j-1 / 2, k}^{n}\right),
$$

где $Z_{j, k}^{n *}-$ скорректированная функция.

Данный алгоритм выполняется в случае, когда $\left(\delta Z_{j-1 / 2, k}^{n} \delta Z_{j+1 / 2, k}^{n}\right)<0$ или $\left(\delta Z_{j+1 / 2, k}^{n} \delta Z_{j+3 / 2, k}^{n}\right)<$ 0. Здесь используются обозначения

$$
\delta Z_{j-1 / 2, k}^{n}=Z_{j}^{n}-Z_{j-1, k}^{n}, \delta Z_{j+1 / 2, k}^{n}=Z_{j+1, k}^{n}-Z_{j, k}^{n}, \delta Z_{j+3 / 2, k}^{n}=Z_{j+2, k}^{n}-Z_{j+1 k}^{n},
$$

где $\kappa$ - коэффициент коррекции.

На границах расчетной области задавались однородные граничные условия первого рода для скорости и однородные граничные условия второго рода для остальных динамических функций [Кутушев, 2003]:

$$
\begin{gathered}
u_{1}(t, N)=0, u_{2}(t, N)=0, \\
\rho_{1}(t, N)=\rho_{1}(t, N-1), \rho_{2}(t, N)=\rho_{2}(t, N-1), \\
e_{1}(t, N)=e_{1}(t, N-1), e_{2}(t, N)=e_{2}(t, N-1), \\
u_{1}(t, 1)=0, u_{2}(t, 1)=0, \\
\rho_{1}(t, 1)=\rho_{1}(t, 2), \rho_{2}(t, 1)=\rho_{2}(t, 2), \\
e_{1}(t, 1)=e_{1}(t, 2), e_{2}(t, 1)=e_{2}(t, 2) .
\end{gathered}
$$

В начальный момент времени компоненты смеси покоились:

$$
u_{1}(0, i)=0, u_{2}(0, i)=0 .
$$

Для искомых функций в моделируемой области течения задавались начальные значения: $e_{1}(0, i)=$ $e_{1}^{0}, e_{2}(0, i)=e_{2}^{0}, \rho_{1}(0, i)=\rho_{1}^{0}, \rho_{2}(0, i)=\rho_{2}^{0}$ в левой половине канала $(\mathrm{x}<\mathrm{L} / 2)$ и в правой половине канала $(\mathrm{x} \geq \mathrm{L} / 2): e_{1}(0, i)=0, e_{2}(0, i)=0, \rho_{1}(0, i)=0, \rho_{2}(0, i)=0$. Численное решение проводилось на равномерной сетке с количеством узлов вдоль оси $x-N=1000$. Шаг по времени вычислялся исходя из условия Куранта-Фридрихса-Леви [Fletcher, 1988]. Алгоритм численного решения системы уравнений математической модели был реализован на языке Fortran. Использованная в работе математическая модель тестировалась сопоставлением результатов моделирования с результатами известных из литературы численных расчетов [Губайдуллин, Тукмаков, 2014], экспериментальными результатами [Нигматулин и др., 2016] и аналитическими решениями [Губайдуллин, Тукмаков, 2014].

3. Результаты расчетов. Начальное давление и температура газа $\mathrm{p}=98 \mathrm{\kappa}$ Па и $\mathrm{T}=293 \mathrm{~K}$, длина канала $-\mathrm{L}=1$ м. Диаметр частиц моделируемого потока газовзвеси составлял $\mathrm{d}=20$ мкм, плотность материала частиц $\rho_{20}=2500 \mathrm{kг} / \mathrm{m}^{3}$. На рис. 1 схематично изображен канал одна часть которого заполнена газом со взвешенными в нем дисперсными частицами, а в другой части которого расположена разряженная среда.

На рис. 2 представлены результаты аналитических [Овсянников, 2003] и численных расчетов скорости однородного газа - кривые 3 и 2 соответственно, также на рисунке изображено пространственное распределение скорости газа при разлете газовзвеси, $\alpha=0.001$. Сопоставление скоростей в аналитическом решении $(\mathrm{u}=731 \mathrm{~m} / \mathrm{c})$ и численном расчете $(\mathrm{u}=561 \mathrm{~m} / \mathrm{c})$ для вязкого газа показывает, что наличие вязкости влияет на скорость спутного потока при истечении газа. Наличие дисперсной компоненты приводит к существенному уменьшению скорости газа - u=319 м/с.

Увеличение объемного содержания дисперсной фазы $(\alpha=0.0001)$ приводит к замедлению истечения газовзвеси в вакуум, в то же время процесс истечения в вакуум газовзвеси с малым объемным содержанием дисперсной фазы $(\alpha=0.00001)$ аналогичен процессу истечения чистого газа - рис. 3.

Скорость газа для численной модели истечения однородного вязкого газа составляет $-\mathrm{u}=561$ м / с для газовзвеси с объемным содержанием $\alpha=0.00001-\mathrm{u}=550 \mathrm{~m} / \mathrm{c}$; для газовзвеси с объемным содержанием $\alpha=0.0001-\mathrm{u}=514 \mathrm{~m} / \mathrm{c}$; для газовзвеси с объемным содержанием $\alpha=0.001-\mathrm{u}=319 \mathrm{м} / \mathrm{c}$ : рис. 4.

Численное моделирование показывает, что при увеличении объемного содержания дисперсной компоненты $(0.0001 \leq \alpha)$ существенно уменьшается скорость истекающего газа. При этом с увеличением объемного содержания дисперсной фазы уменьшается интенсивность скоростного скольжения фаз - рис. 5, также происходит уменьшение скорости движения дисперсной компоненты смеси рис. 6. 


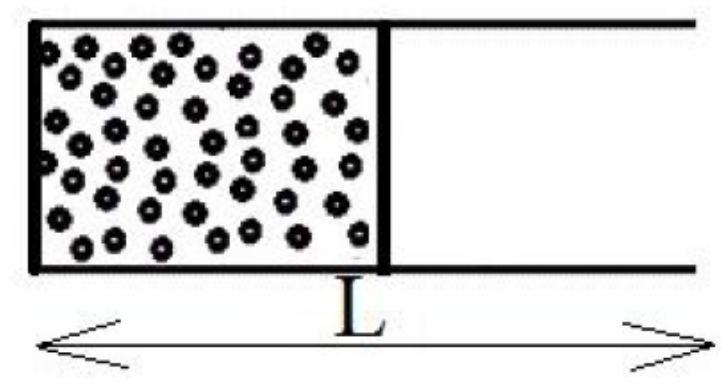

Рис. 1. Схематичное изображение моделируемого канала

Fig. 1. Schematic representation of the simulated channel

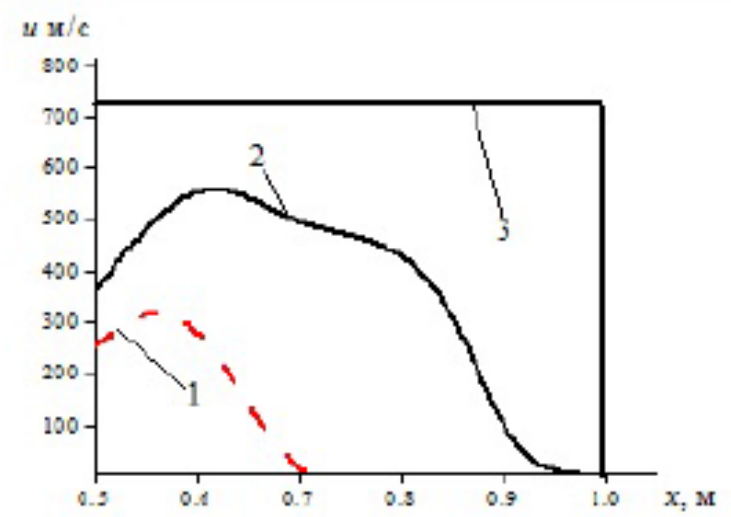

Рис. 2. Пространственное распределение скорости газа при разлете в вакуум газовзвеси с объемным содержанием твердой фазы $\alpha=0.0001$ - кривая 1 ; разлете в вакуум вязкого газа - кривая 2 ; аналитическое решение для невязкого газа - кривая 3 . Момент времени $\mathrm{t}=0.3 \mathrm{мc}$

Fig. 2. Spatial distribution of gas velocity during expansion of a gas suspension with a volumetric solid phase content of $\alpha=0.0001$ - curve 1; expansion into a vacuum of viscous gas - curve 2; analytical solution for an inviscid gas - curve 3 . Moment of time.t $=0.3 \mathrm{~ms}$

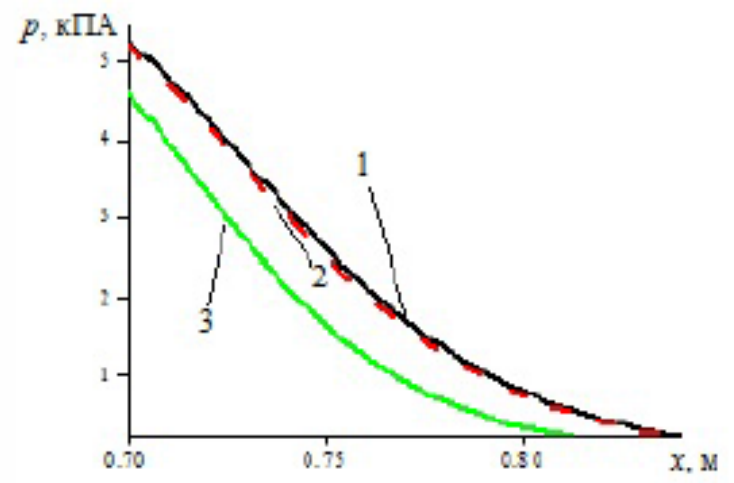

Рис. 3. Пространственные распределения давления газа при разлете в вакуум однородного вязкого газа кривая 1 ; разлете в вакуум вязкого газа с дисперсной компонентой $(\alpha=0.00001)$ - кривая 2 ; разлете в вакуум вязкого газа с дисперсной компонентой $(\alpha=0.0001)$ - кривая 3 . Момент времени $\mathrm{t}=0.3$ мс

Fig. 3. Spatial distributions of gas pressure during expansion into a vacuum of a homogeneous viscous gas curve 1; expansion of a viscous gas with a dispersed component into vacuum $(\alpha=0.00001)$ - curve 2 ; expansion of a viscous gas with a dispersed component $(\alpha=0.0001)$ - curve 3 into vacuum. Moment of time $-\mathrm{t}=0.3 \mathrm{~ms}$ 


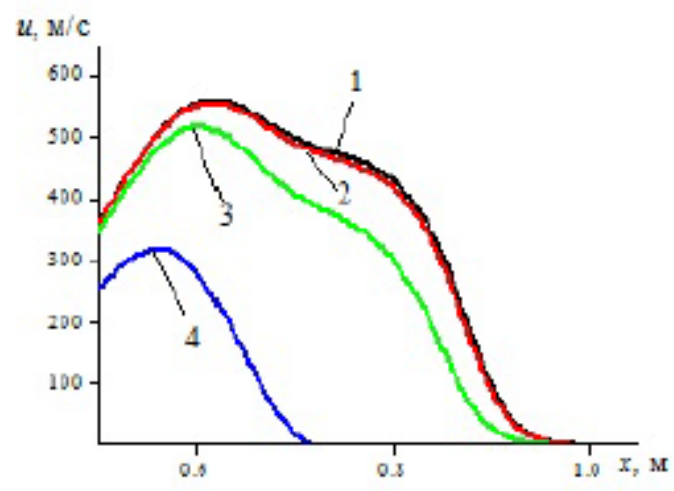

Рис. 4. Пространственное распределение скорости газа при разлете в вакуум однородного вязкого газа кривая 1 ; разлете в вакуум вязкого газа с дисперсной компонентой $(\alpha=0.00001)$ - кривая 2 ; разлете в вакуум вязкого газа с дисперсной компонентой $(\alpha=0.0001)$ - кривая 3 ; разлете в вакуум вязкого газа $\mathrm{c}$ дисперсной компонентой $(\alpha=0.001)$ - кривая 4 . Момент времени $\mathrm{t}=0.3 \mathrm{мc}$

Fig. 4. Spatial distribution of gas velocity during the expansion of a homogeneous viscous gas into a vacuum curve 1; expansion into a vacuum of a viscous gas with a dispersed component $(\alpha=0.00001)$ - curve 2 ; expansion of a viscous gas with a dispersed component $(\alpha=0.0001)$ - curve 3 into vacuum; expansion of a viscous gas with a dispersed component $(\alpha=0.001)$ - curve 4 into vacuum. Moment of time.t $=0.3 \mathrm{~ms}$

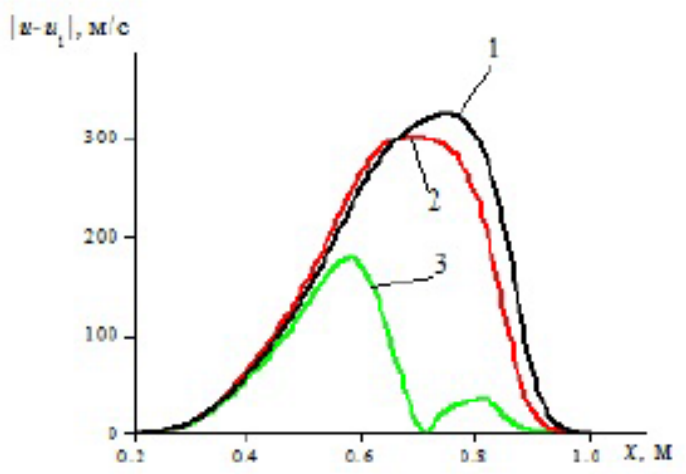

Рис. 5. Пространственное распределение интенсивности скоростного скольжения компонент смеси при разлете в вакуум вязкого газа с дисперсной компонентой $(\alpha=0.00001)$ - кривая 1 ; разлете в вакуум вязкого газа с дисперсной компонентой $(\alpha=0.0001)$ - кривая 2 ; разлете в вакуум вязкого газа с дисперсной компонентой $(\alpha=0.001)$ - кривая 3 . Момент времени $\mathrm{t}=0.3 \mathrm{мc}$

Fig. 5. The spatial distribution of the intensity of the velocity slip of the mixture components during the expansion of a viscous gas with a dispersed component into vacuum $(\alpha=0.00001)$ - curve 1 ; expansion of a viscous gas with a dispersed component into vacuum $(\alpha=0.0001)$ - curve 2 ; expansion of a viscous gas with a dispersed component $(\alpha=0.001)$ - curve 3 into vacuum. Moment of time $-\mathrm{t}=0.3 \mathrm{~ms}$

Выявленные закономерности можно объяснить тем, что при прочих неизменных параметрах дисперсной компоненты смеси, увеличение объемного содержания дисперсной фазы газовзвеси приводит к увеличению интенсивности межфазного взаимодействия, большим потерям кинетической энергии истекающего в вакуум газа. Вследствие чего скоростные параметры компонент смеси имеют меньшее отличие и интенсивность скоростного скольжения уменьшается. В газовзвесях с меньшим содержанием дисперсной компоненты влияние дисперсной составляющей смеси на динамику газа незначительное, по сравнению с газовзвесями, в которых масса несущей и дисперсной компонент близки. Газовая компонента смеси разгоняется до больших скоростей, чем газовая компонента в газовзвесях с большим объемным содержанием дисперсной фазы. За счет меньших потерь в межфазном взаимодействии скорости истечения газа в газовзвеси с малыми объемными содержаниями дисперсной компоненты близки к скорости истечения чистого газа. При этом скорость дисперсной компоненты существенно меньше, чем у газа, что приводит к скоростному скольжению большей величины. 


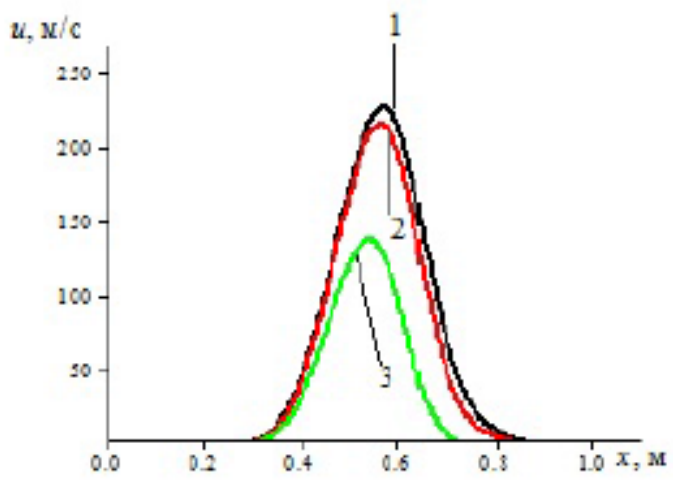

Рис. 6. Пространственное распределение скорости дисперсной компоненты смеси при разлёте в вакуум вязкого газа с дисперсной компонентой $(\alpha=0.00001)$ - кривая 1 ; разлёте в вакуум вязкого газа с дисперсной компонентой $(\alpha=0.0001)$ - кривая 2 ; разлёте в вакуум вязкого газа с дисперсной компонентой $(\alpha=0.001)$ - кривая 3. Момент времени $\mathrm{t}=0.3 \mathrm{мc}$

Fig. 6. Spatial velocity distribution of the dispersed component of the mixture during the expansion of a viscous gas with a dispersed component $(\alpha=0.00001)$ - curve 1 ; expansion of a viscous gas with a dispersed component $(\alpha=0.0001)$ - curve 2 into vacuum; expansion of a viscous gas with a dispersed component $(\alpha=0.001)$ - curve 3 into vacuum. Moment of time $-\mathrm{t}=0.3 \mathrm{~ms}$

Выводы. Вычислительные эксперименты выявили отличия процессов истечения в вакуум однородного газа и неоднородной среды. Математическое моделирование продемонстрировало, что наличие дисперсной фазы оказывает существенное влияние на истечение газа в вакуум. Также было определено, что при объёмных содержаниях дисперсной компоненты смеси больших $\alpha=0.0001$ влияние дисперсной компоненты смеси становится существенным. Интенсивность межфазного скоростного скольжения обратно пропорциональна величине объёмного содержания дисперсной компоненты смеси. Увеличение объёмного содержания дисперсной компоненты смеси приводит к уменьшению скорости движения несущей и дисперсной компонент смеси, вследствие большего межфазного взаимодействия, в результате уменьшается интенсивность межфазного скоростного скольжения.

\section{Список литературы}

1. Арсланова С. Н., Тукмаков А. Л., Тонконог В. Г. 2016. Волновая коагуляция полидисперсной газовзвеси в технологии газификации и криостатирования сжиженного природного газа. Акустический журнал. 62(1): 125-131.

2. Вараксин А. Ю. 2014. Кластеризация частиц в турбулентных и вихревых двухфазных потоках. Теплофизика высоких температур. 52(5): 777-796.

3. Губайдуллин Д. А., Тукмаков Д.А. 2014. Исследование динамики двухкомпонентного газа с пространственно разделенными в начальный момент компонентами. Известия вузов. Проблемы энергетики, (3-4): 38-43.

4. Губайдуллин Д. А., Тукмаков Д.А. 2014. Численное исследование эволюции ударной волны в газовзвеси с учетом неравномерного распределения частиц. Математическое моделирование, 26(10): 109-119.

5. Кутушев А. Г. 2003. Математическое моделирование волновых процессов в аэродисперсных и порошкообразных средах. СПб.: Недра, 284 с.

6. Музафаров И. Ф., Утюжников С. В. 1993. Применение компактных разностных схем к исследованию нестационарных течений сжимаемого газа. Математическое моделирование, 5(3):74-83.

7. Нигматулин Р. И. 1987. Динамика многофазных сред. Ч. 1 М.: Наука, 464 с.

8. Нигматулин Р. И., Губайдуллин Д.А., Тукмаков Д.А. 2016. Ударно-волновой разлет газовзвесей. Доклады академии наук, 466(4): 418-421.

9. Овсянников Л. В. 2003. Лекции по основам газовой динамики. Москва: Издательство Института компьютерных исследований, 336 с. 
10. Пахомов М. А., Терехов В. И. 2018. Влияние испарения капель на структуру течения и тепломассобмен в ограниченном закрученном газокапельном потоке за его внезапным расширение. Теплофизика и аэромеханика, 6: 865-875.

11. Садин Д. В. 2016. TVD-схема для жестких задач волновой динамики гетерогенных сред негиперболического неконсервативного типа. Журнал вычислительной математики и математической физики, 56(12): 2098-2109.

12. Тукмаков А. Л., Тукмаков Д. А. 2018. Генерация акустического возмущения движущейся заряженной газовзвесью. Инженерно-физический журнал, 91(5): 1207-1203.

13. Тукмаков А. Л., Кашапов Н. Ф., Тукмаков Д. А., Фазлыйяхматов М. Г. 2018. Процесс осаждения заряженной полидисперсной газовзвеси на поверхность пластины в электрическом поле. Теплофизика высоких температур, 56(4): 498-502.

14. Тукмаков Д. А. 2019. Численное моделирование отражения ударной волны в газовой взвеси для различных параметров дисперсной компоненты запыленной среды. Научные ведомости Белгородского государственного университета. Серия: Математика. Физика. 51(4): 565-576.

15. Федоров А. В., Фомин В. М., Хмель Т. А. 2015. Волновые процессы в газовзвесях частиц металлов. Новосибирск, 301 с.

16. Федоров А. В., Михайлов А. Л., Финюшин С. А., Калашников Д. А., Чудаков Е. А., Бутусов Е. И., Гнутов И. С. 2016. Регистрация параметров множественного откола и внутренней структуры облака частиц при ударноволновом нагружении металлов. Журнал экспериментальной и теоретической физики, 149(4): 792-795.

17. Azanov G. M., Osiptsov A. N. 2017. The efficiency of one method of machineless gasdynamic temperature stratification in a gas flow. International Journal of Heat and Mass Transfer, 106: 1125-1133.

18. Fletcher C. 1988. Computation Techniques for Fluid Dynamics, Berlin: Springer-Verlang, 502 p.

19. Garcia-Trinanes P., Seville J., Ansart R., Benoit H., Leadbeater T., Parker D. 2018. Particle motion and heat transfer in an upward-flowing dense particle suspension: Application in solar receivers Chemical Engineering Science, 177: 313-322.

20. Imran M., Khan H., Joardder M., Kumar C., Karim M. 2018. Multiphase porous media modelling: A novel approach to predicting food processing performance. Critical Reviews in Food Science and Nutrition 58(4): 528-546.

21. Lopez I., Benoit H., Gauthier D., Sans J., Guillot E., Mazza G., Flamanta G. 2016. On-sun operation of a $150 \mathrm{kWth}$ pilot solar receiver using dense particle suspension as heat transfer fluid Solar Energy, 137: $463-476$.

22. Lopez I., Benoit H., Gauthier D., Sans J., Guillot E., Mazza G., Flamanta G. 2016. On-sun operation of a $150 \mathrm{kWth}$ pilot solar receiver using dense particle suspension as heat transfer fluid Solar Energy, 137: 463-476.

23. Mehrabadi M., Tenneti S., Garg R., Subramaniam S. 2015. Pseudo-turbulent gas-phase velocity fluctuations in homogeneous gas-solid flow: fixed particle assemblies and freely evolving suspensions. Journal of Fluid Mechanics, 770: 210-246.

24. Saikat S., Meheboob A. 2017. Revisiting ignited-quenched transition and the non-Newtonian rheology of a sheared dilute gas-solid suspension. Journal of Fluid Mechanics, 833: 206-246.

25. Teklaya A., Chungen Y., Lasse R., Kohler L. 2015. Experimental and modeling study of flash calcination of kaolinite rich clay particles in a gas suspension calciner. Applied Clay Science, 103: 10-19.

26. Tukmakov A. L., Kashapov N.F., Tukmakov D.A., Fazlyyakhmatov M.G. 2018. Numerical modeling of the powder materials spraying. IOP Conference Series: Materials Science and Engineering, https://iopscience.iop.org/article/10.1088/1757-899X/412/1/012076.

27. Zhengbiao P., Behdad M., Elham D. 2017. Suspension stability of slurry Taylor flow: A theoretical analysis.Chemical Engineering Science, 174: 459-471. 
28. Zhuoqing A. Jesse Z. 2019. Correlating the apparent viscosity with gas-solid suspension flow in straight pipelines. Powder Technology, 345: 346-351.

\section{References}

1. Arslanova S. N., Tonkonog V. G., Tukmakov A. L. 2016. Wave coagulation of a polydisperse gas suspension in the technology of gasification and Cryostatting of liquefied natural gas. Acoustical Physics. 62(1): 125-131.

2. Varaksin A. Y. 2014. Clusterization of particles in turbulent and vortex two-phase flows. High Temperature, 52(5): 752-769.

3. Gubaidullin D. A., Tukmakov D. A. 2014. A study of the dynamics of a two-component gas with components spatially separated at the initial moment. News of higher educational institutions. energy issues, (3-4): 38-43. (in Russian)

4. Gubajdulli D. A., Tukmakov D. A. 2015. Numerical investigation of the evolution of a shock wave in a gas suspension with consideration for the nonuniform distribution of the particles. Mathematical Models and Computer Simulations, 7(3): 246-253.

5. Kutushev A.G. 2003. Matematicheskoe modelirovanie volnovyh processov v aerodispersnyh i poroshkoobraznyh sredah.SPb.: Nedra, 284 p.

6. Muzafarov I. F., Utyuzhnikov S. V. 1993. Application of compact difference schemes to the study of unsteady flows of a compressible gas. Mathematical Modeling, 5 (3): 74-83. (in Russian)

7. Nigmatulin R. I. 1987. Dinamika mnogofaznyh sred Ch.1 M.: Nauka, 464 p.

8. Nigmatulin R. I., Gubaidullin D. A., Tukmakov D. A. 2016. Shock Wave Dispersion of Gas-Particle Mixtures. Doklady Physics, 61(2): 70-73.

9. Ovsyannikov L. V. 2003. Lekcii po osnovam gazovoj dinamiki. Moskva, Izdatel'stvo Instituta komp'yuternyh issledovanij, $336 \mathrm{p}$.

10. Pakhomov M. A., Terekhov V. I. 2018. Effect of droplet evaporation on the flow structure and heat and mass transfer in a confined swirling gas-droplet flow downstream of a tube sudden expansion. Thermophysics and Aeromechanics, 25(6): 833--843.

11. Sadin D. V. 2016. TVD scheme for stiff problems of wave dynamics of heterogeneous media of nonhyperbolic nonconservative type. Computational Mathematics and Mathematical Physics, 56(12): 2068-2078.

12. Tukmakov A. L., Tukmakov D. A. 2018. Generation of Acoustic Disturbances by a Moving Charged Gas Suspension. Journal of Engineering Physics and Thermophysics, 91(5): 1141-1147.

13. Tukmakov A. L., Kashapov N. F., Tukmakov D. A., Fazlyyyakhmatov M. G. 2018. Process of the Deposition of Charged Polydisperse Gas Suspension on the Plate Surface in an Electrical Field. High Temperature, 56(4): 481-485.

14. Tukmakov D. A. 2019. Numerical modeling of reflection of a shock wave in a gas suspension for various parameters of a dispersed component of a dusted medium. Belgorod State University Scientific Bulletin. Mathematics. Physics. 51(4): 565-576 (in Russian).

15. Fedorov A. V., Fomin V. M., Hmel T. A. 2015. Volnovye processy v gazovzvesyah chastic metallov. Novosibirsk, $301 \mathrm{p}$.

16. Fedorov A. V., Mikhailov A. L., Finyushin S. A., Kalashnikov D. A., Chudakov E. A., Butusov E. I., Gnutov I. S. 2016. Detection of the multiple spallation parameters and the internal structure of a particle cloud during shock-wave loading of a metal. Journal of Experimental and Theoretical Physics, 122(4): 685-688.

17. Azanov G. M., Osiptsov A. N. 2017. The efficiency of one method of machineless gasdynamic temperature stratification in a gas flow. International Journal of Heat and Mass Transfer, 106: $1125-1133$.

18. Fletcher C. 1988. Computation Techniques for Fluid Dynamics, Berlin: Springer-Verlang, 502 p. 
19. Garcia-Trinanes P., Seville J., Ansart R., Benoit H., Leadbeater T., Parker D. 2018. Particle motion and heat transfer in an upward-flowing dense particle suspension: Application in solar receivers Chemical Engineering Science, 177: 313-322.

20. Imran M., Khan H., Joardder M. , Kumar C., Karim M. 2018. Multiphase porous media modelling: A novel approach to predicting food processing performance. Critical Reviews in Food Science and Nutrition 58(4): 528-546.

21. Lopez I., Benoit H., Gauthier D., Sans J., Guillot E., Mazza G., Flamanta G. 2016. On-sun operation of a $150 \mathrm{kWth}$ pilot solar receiver using dense particle suspension as heat transfer fluid Solar Energy, 137: 463-476.

22. Lopez I., Benoit H., Gauthier D., Sans J., Guillot E., Mazza G., Flamanta G. 2016. On-sun operation of a $150 \mathrm{kWth}$ pilot solar receiver using dense particle suspension as heat transfer fluid Solar Energy, 137: 463-476.

23. Mehrabadi M., Tenneti S., Garg R., Subramaniam S. 2015. Pseudo-turbulent gas-phase velocity fluctuations in homogeneous gas-solid flow: fixed particle assemblies and freely evolving suspensions. Journal of Fluid Mechanics, 770: 210-246.

24. Saikat S., Meheboob A. 2017. Revisiting ignited-quenched transition and the non-Newtonian rheology of a sheared dilute gas-solid suspension. Journal of Fluid Mechanics, 833: 206-246.

25. Teklaya A., Chungen Y., Lasse R., Kohler L. 2015. Experimental and modeling study of flash calcination of kaolinite rich clay particles in a gas suspension calciner. Applied Clay Science, 103: 10-19.

26. Tukmakov A. L., Kashapov N. F., Tukmakov D. A., Fazlyyakhmatov M.G. 2018. Numerical modeling of the powder materials spraying. IOP Conference Series: Materials Science and Engineering, https://iopscience.iop.org/article/10.1088/1757-899X/412/1/012076.

27. Zhengbiao P., Behdad M., Elham D. 2017. Suspension stability of slurry Taylor flow: A theoretical analysis. Chemical Engineering Science, 174: 459-471.

28. Zhuoqing A. Jesse Z. 2019. Correlating the apparent viscosity with gas-solid suspension flow in straight pipelines. Powder Technology, 345: 346-351.

Тукмаков Дмитрий Алексеевич - кандидат физико-математических наук, старший научный сотрудник института механики и машиностроения - обособленного структурного подразделения Федерального государственного бютжетного учреждения науки «Федеральный иследовательский центр «Казанский научный центр Российской академии наук»

ул. Лобачевского, д. 2/31, г. Казань, Россия, 420111

E-mail: tukmakovDA@imm.knc.ru 